\title{
A Novel Method to Reduce Peak-To-Average Power Ratio (PAPR) In Multicarrier Code Division Multiple Access (MC-CDMA)
}

\author{
Mohammed Ahmed El-Absi and Mohammed Tawfik Hussein
}

\begin{abstract}
This paper proposes an efficient peak-to-average power ratio (PAPR) reduction method for multicarrier code division multiple access (MC-CDMA) signals by decreasing the possibility of peaks of the subcarriers signals to occur at the same time. The achieved results confirm that the proposed method is capable of reducing the PAPR significantly and, therefore, of improving error performance of the system.
\end{abstract}

Index Terms-CDMA, MC-CDMA, OFDM, PAPR.

\section{INTRODUCTION}

Mobile radio communication systems are required to provide high-quality multimedia services to mobile users. To meet these requirements, modern mobile systems must be able to support high capacity and variable bit rate transmission with bandwidth efficiency to conserve the limited spectrum resource. Recently, MC-CDMA gained a lot of attention for future generation of wireless communication systems. MC-CDMA is a hybrid combination of two access techniques, orthogonal frequency division multiplexing (OFDM) and Code Division Multiple Access (CDMA) in hopes to take advantage of two techniques. CDMA is a multiplexing technique where different users share the same frequency band at the same time. Recently, CDMA technique has been considered a candidate that requires fast and reliable data transfer to support multimedia services, video conferencing and more applications. $3 \mathrm{G}$ systems will operate at $2 \mathrm{GHz}$ region thus able to provide up to $2 \mathrm{Mbps}$. On the other hand, OFDM is considered a solution for the selectivity of the channel. This gives the ability for transmitting high data rate in fading environment $[1,2]$.

MC-CDMA poses high peak-to-average power ratio (PAPR) problem, as a consequence of independently modulated carriers. To be able to handle these occasional large peaks, a high-power amplifier with linear characteristics over a large dynamic range is required. This brings disadvantages like an increased complexity of the $\mathrm{ADC}$ and DAC converters and a reduced efficiency of the power amplifier because the power consumption of a high-power amplifier largely depends on its peak power output rather than the average power output [3].

On the other hand, if the high power amplifier does not handle high peaks (i.e., behaves nonlinearly), the resulting signal clipping will cause out-of-band radiation and

Manuscript received March 25, 2010. degradation of the performance [4].

In literature, a variety of PAPR reduction techniques have been proposed. Clipping the OFDM signal in the transmitter [5], the tone reservation method [6], partial transmit sequences algorithm (PTS) $[7,8]$, selected mapping algorithm (SLM) [9], pulse shaping [10], random phase updating [11], subblock phase weighting scheme [12], linear scaling [13] and precoding [14]. All of these techniques done for OFDM signals only and little bit of them take MC-CDMA signal in consideration.

In this paper, we propose new technique to change the location of subcarriers to symbols that produces the smallest PAPR. This paper is organized as follows. In section II we define the PAPR of an MC-CDMA signal, and discuss related issues. In section III, the proposed method is outlined. In section IV, the system model is shown. In section V the performance of the proposed method is presented. Section VI is devoted to discussions and conclusions.

\section{MC-CDMA SIGNAL AND PAPR MODELS}

The time domain MC-CDMA signal with interval $0<t<T_{s}$ and defined by [15]:

$$
x(t)=\frac{1}{\sqrt{N}} \sum_{n=0}^{N-1} X_{n} e^{j 2 \pi f_{n} t}
$$

Where $T s$ chip period, $N=P G_{M C}$ subcarriers, $G_{M C}$ denotes the processing gain, $P$ denotes the number of symbols in each block.

Vector $\mathrm{X}$ is defined as:

$\mathrm{X}=\left[\boldsymbol{D}_{0}\left[c_{1}^{k}, c_{2}^{k}, \ldots, c_{c_{M C}}^{k}\right], D_{1}\left[c_{1}^{k}, c_{2}^{k}, \ldots, c_{c_{M C}}^{k}\right], \ldots, D_{P-1}\left[c_{1}^{k}, c_{2}^{k}, \ldots, c_{c_{M C}}^{k}\right]\right]$ $\mathrm{D}$ is a vector that expresses the data of the ith block of size $\mathrm{P}$ after serial to parallel conversion, $\mathrm{D}$ defined as:

$$
\mathrm{D}=\left[D_{0}, D_{1}, \ldots \ldots, D_{P-1}\right]
$$

$\mathrm{C}$ is a vector that expresses the spreading code for the $k t h$ user.

$f_{n}$ is the $n$th subcarrier frequency that equals $n \Delta f$. Note that the subcarrier frequency separation is $\Delta f=1 / T_{s}$.

PAPR is defined as the ratio of the peak instantaneous power and the average power, this mathematically can be expressed as $[15,16]$ :

$$
\text { PAPR }=\frac{\max _{0<t<T_{s}}|x(t)|^{2}}{E\left\{|x(t)|^{2}\right\}}
$$




\section{PRoposed METHOD FOR PAPR REDUCTION}

In conventional MC-CDMA, $n$th chip is assigned to a subcarrier with frequency $n \Delta f$, where $\Delta f$ is the subcarrier frequency separation and $n=0,1,2, \ldots ., N-1$. Our proposed technique is to change the location of the default set of subcarriers before assigning them to chips.

This means that it is not necessary for the set of subcarriers to be arranged in ascending order. Based on this, a search for suitable sets of subcarriers is performed with the objective of finding the set that produces the lowest PAPR.

To illustrate the proposed method procedure above, let us assume a default MC-CDMA system, with block size $\mathrm{P}$ equal to $32, G_{M C}=31$, this mean that the number of subcarriers $N=992$. A data chip period $T_{s}=1$, this means that $\Delta f=1$. Therefore, the default set of subcarriers is specified as $\{0,1,2, \ldots \ldots \ldots . . ., 991\}$. If we shuffle this set we may end, for example with the set of subcarriers $\{3,502,100,992, \ldots \ldots \ldots . ., 5\}$ that minimizes the PAPR. Using this criterion, we maintain orthogonality between subcarriers; because the minimum separation between subcarriers is preserved. In addition, the bandwidth is not changed by using the proposed method, and no bandwidth expansion is incurred.

Comparing the default and shuffled sets of subcarriers in the example above reveals that the process of assigning subcarriers to data symbols is no longer a pure inverse fourier transform (IFFT) operation. The IFFT block has to be replaced with another block. This also means that the FFT block in the receiver should be changed. We can replace the discrete Fourier transform by what may be called the "generalized discrete Fourier transform" (GDFT) block.

\section{SYSTEM MODEL}

Two blocks diagram of the modified MC-CDMA transmitter and receiver with $K_{u}$ active users are shown in figures 1 and 2 respectively.

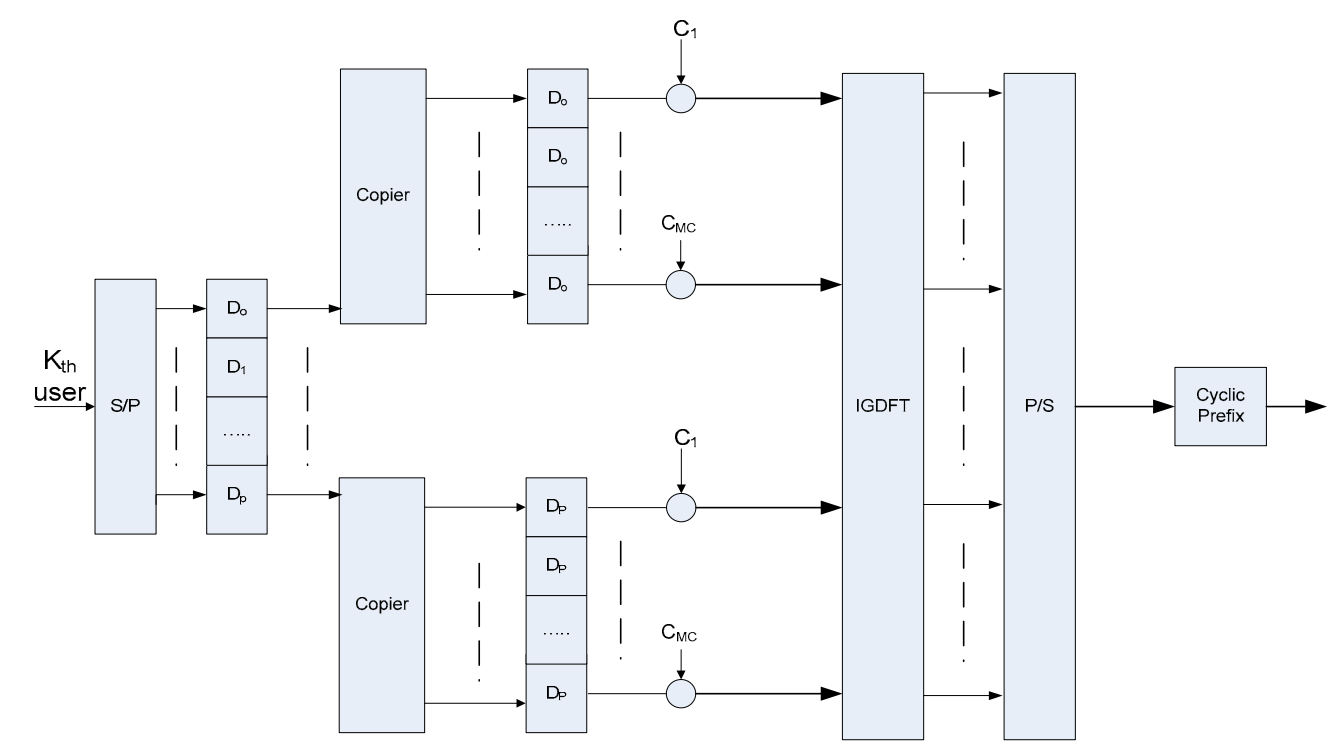

Fig. 1: MC-CDMA Transmitter

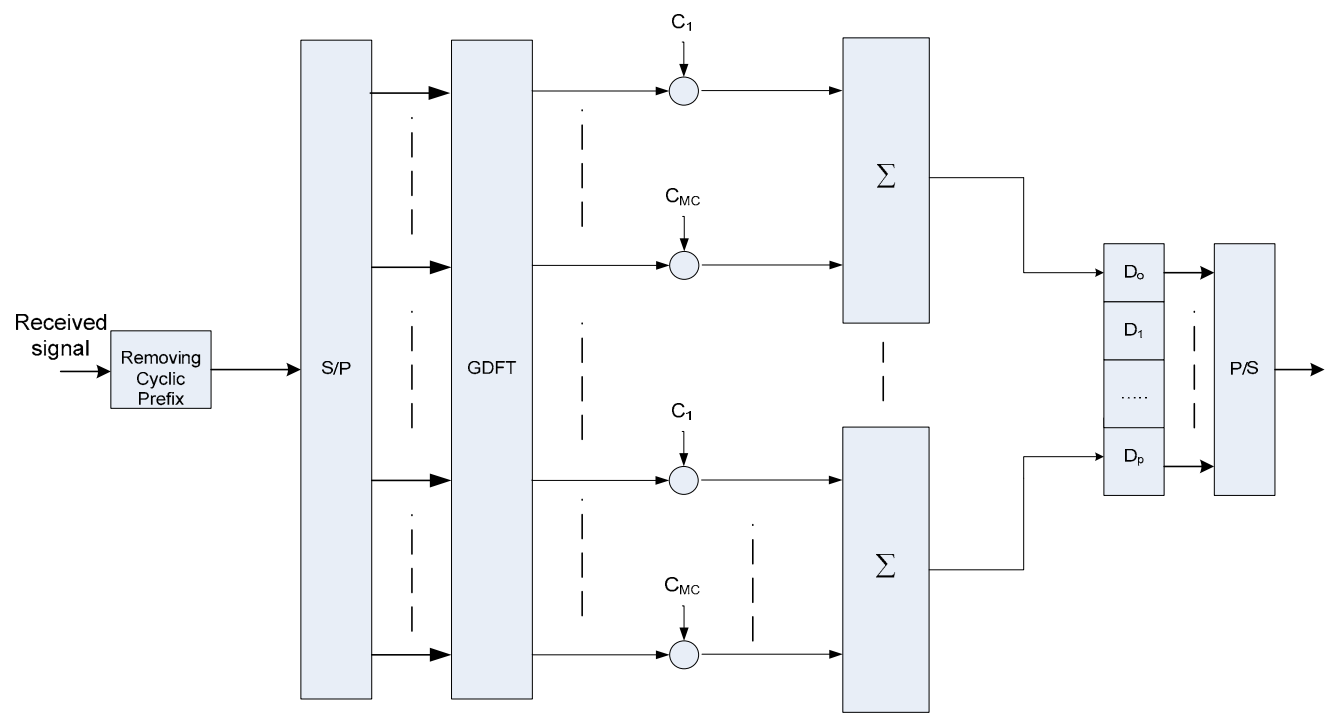

Fig. 2: MC-CDMA receiver

The MC-CDMA transmitter configuration for the $k t h$ user is shown in figure 1. After serial-to-parallel conversion of $\mathrm{P}$ symbols, the pth symbol of the kth user in the ith block is spread by user-specific spreading code. $c_{k}(t)=\left[c_{1}^{k}, c_{2}^{k}, \ldots, c_{G_{M C}}^{k}\right]$ is the spreading code of the $k t h$ user 
in the frequency domain, $G_{M C}$ denotes the processing gain.

Gold code is used. Each chip is mapped to one subcarrier. In total, we have $N=P G_{M C}$ subcarriers. The users are separated by different codes. All data corresponding to the total number of subcarriers are modulated by an IGDFT. The order of subcarriers used in the IGDFT are chosen to minimize PAPR.

The data is converted back into serial data. Then guard interval is inserted to combat ISI. Finally the signal is digital to analog converted and unconverted for transmission. Rayleigh distribution for the fading channel model was assumed.

The MC-CDMA receiver configuration for the kth user is shown in Figure 2. The received signal is first downconverted, and the cyclic prefix is removed. Then, the data is serial-to-parallel converted. After that, the signal is transformed using GDFT to be ready for despreading. In simulation we consider other users at different SNR. Then the data is ready to be detected. MatLab was selected as the simulation tool to implement the modified MC-CDMA system.

\section{Performance Comparisons}

We use computer simulations to evaluate the performance of the proposed PAPR reduction techniques. To measure the performance for the proposed techniques, we use the PAPR complementary cumulative density function (CCDF) and the PAPR histogram. The CCDF displays the probability of the PAPR exceeding a certain value, while the histogram displays the distribution of PAPR values.

Ten thousand blocks of size $\mathrm{P}=32$ were generated randomly to obtain the CCDFs. Every symbol from the $\mathrm{P}$ symbol is spread using spreading code. Then each chip is modulated using one subcarrier from $N=P G_{M C}$ subcarriers. BPSK data modulation was assumed.

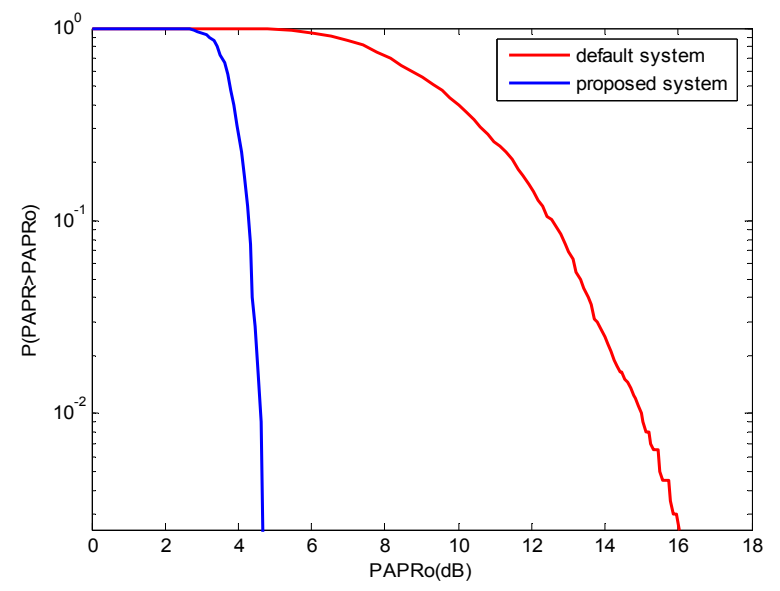

Fig.3: PAPR CCDF: BPSK Data Modulation, $\mathrm{P}=32$.

Figure 3 shows PAPR CCDFs for the default set of subcarrier frequencies that were used in conventional MC-CDMA and the new set of subcarrier frequencies that were used in modified MC-CDMA. We clearly note that the new set of subcarriers gives lower PAPR than the default set of subcarriers. Great improvement has occurred.

For the same case presented above figure 4 shows the histogram for the default MC-CDMA system that uses the default set of subcarriers, and it displays the number of occurrences of different values of PAPR. We observe large values of PAPR that occur with high probability. The majority of PAPR values for the default set fall in the region between

$5 \mathrm{~dB}$ and $15 \mathrm{~dB}$ and concentrate with high probability between $8 \mathrm{~dB}$ and $12 \mathrm{~dB}$.

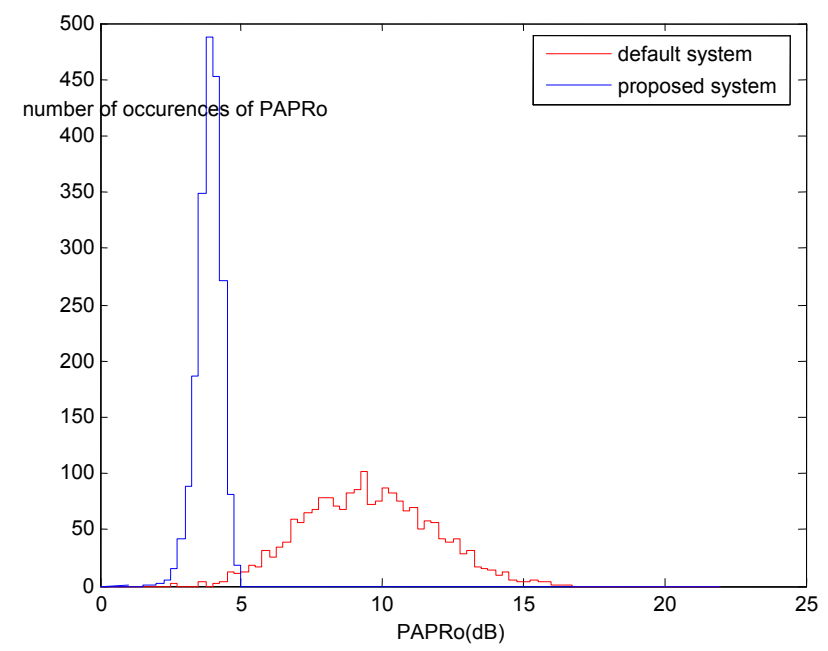

Fig. 4: PAPR Histogram: BPSK Data Modulation, $P=32$

Figure 4 also shows the histogram for the modified MC-CDMA system that uses the new set of subcarriers. We observe that a majority of PAPR values for the new set fall in the region between $2 \mathrm{~dB}$ and $5 \mathrm{~dB}$, and that large PAPR values occur with very small probability. We also note that PAPR values concentrate with high probability between $3.5 \mathrm{~dB}$ and $4.5 \mathrm{~dB}$.

To further test the proposed method, we consider MC-CDMA system that uses 16-QAM data modulation with a block size $\mathrm{P}$ equal to 32 . Figure 5 shows PAPR CCDFs for the default set of subcarrier frequencies that were used in conventional MC-CDMA and the new set of subcarrier frequencies that used in modified MC-CDMA. Great improvement has occurred for the shuffled set of subcarriers. The findings in figure 5 is confirmed in figure 6 which shows the histogram for the modified MC-CDMA system compared with the conventional MC-CDMA. We observe that a majority of PAPR values for the shuffled set fall in the region smaller than the system uses default set of subcarriers.

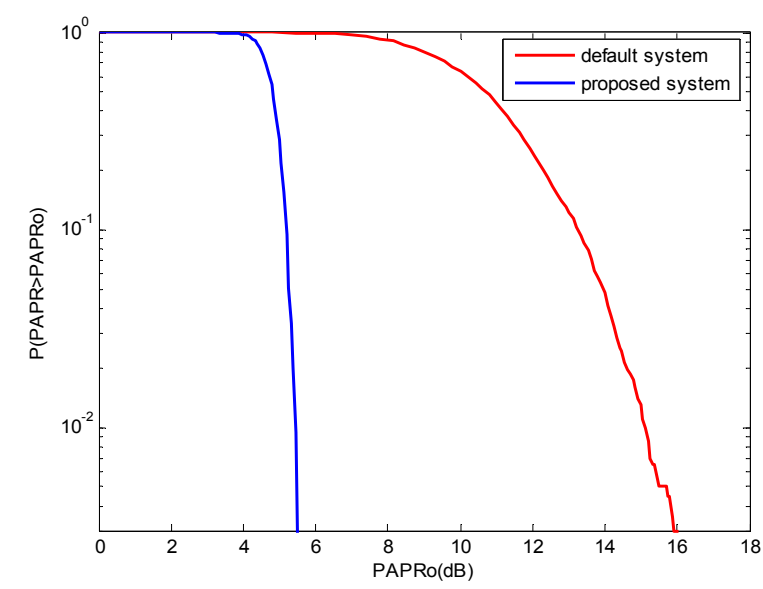

Fig. 5: PAPR CCDF: 16-QAM Data Modulation, $P=32$. 


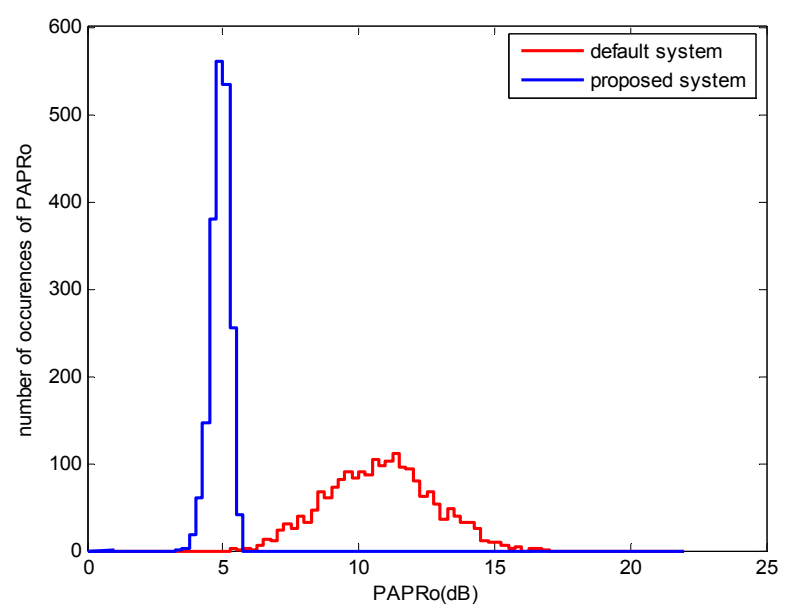

Fig. 6: PAPR Histogram: 16-QAM Data Modulation $P=32$

\section{CONCLUSION}

Recently, MC-CDMA gains a lot attention for future generation of wireless communication systems. New technique has been proposed to reduce PAPR in MC-CDMA systems. It depends on changing the location of subcarriers to obtain a new set that is able to reduce PAPR. The new technique reduced PAPR efficiently. The results of this research confirmed that many related applications in the area of wireless communications can take advantage of the proposed novel method such as Broadband Multi-User Communications, WLANs and Broadcasting.

\section{REFERENCES}

[1] G. Girod, E. James, "A Detailed Study on Multi-Carrier Code Division Multiple Access (MC-CDMA)", Michigan Technological University, 2003.
[2] S. Hara, R. Prasad, "Overview Of Multi-carrier CDMA", IEEE Communication Magazine, Vol. 35, no. 12, pp. 126-133, 1997.

[3] B. Krongold, D. Jones, "PAR reduction in OFDM via active constellation extension", IEEE Transaction Broadcast., Vol. 49, no. 3, pp. 258-268, 2003.

[4] M. Pauli, H. Kuchenbecker, "Minimization Of The Inter-modulation Distortion Of A Nonlinearly Amplified OFDM Signal", Wireless Personal Communications, Vol. 4, no. 1, pp. 93 - 101, 1997.

[5] H. Ochiai, H. Imai, "On clipping for peak power reduction of OFDM signals" IEEE Global Telecommunications Conference, Vol. 2, pp. 731-735, 2000.

[6] M. Sharif, B. H. Khalaj, "Peak to mean envelope power ratio of oversampled OFDM signals: An analytical approach," IEEE International Conference on Communications, Vol. 5, pp. 1476-1480, 2001.

[7] J. Tellado, Multicarrier Modulation With Low PAR: Applications To DSL And Wireless, Kluwer International Series In Engineering And Computer Science, Kluwer Academic Publishers, 2000

[8] A. D. S. Jayalath, C. Tellambura, "Adaptive PTS approach for reduction of peak-to-average power ratio of OFDM signal," Electronics Letters, Vol. 36, no. 14, pp. 1226-1228, 2000.

[9] A. D. S. Jayalath, C. Tellambura, H. K. Wu, "Reduced complexity PTS and new phase sequences for SLM to reduce peak to average power ratio of OFDM", IEEE Vehicular Technology Conference, Vol. 3, pp. 1914-1917, 2000.

[10] S. B. Slimane, "Peak-to-average power ratio reduction of OFDM signals using pulse shaping" IEEE Global Telecommunications Conference, Vol. 3, pp. 1412-1416, 2000

[11] H. Nikookar, K. S. Lidsheim, "PAPR reduction of OFDM by random phase updating", IEEE International Symposium on Personal, Indoor and Mobile Radio Communications, Vol. 2, pp. 814-818, 2002.

[12] H. G. Ryu, K. J. Youn, "A new PAPR reduction scheme: SPW (subblock phase weighting)", IEEE Transactions On Consumer Electronics, Vol. 48, No. 1, pp. 81-89, 2002.

[13] H. Ochiai, "Performance analysis of peak power and band-limited OFDM system with linear scaling", IEEE Transactions on Wireless Communications, vol. 2, no. 5, pp. 1055-1065, 2003.

[14] S. B. Slimane, "Reducing the peak-to-average power ratio of OFDM signals through precoding," IEEE Transactions On Vehicular Technology, Vol. 56, No. 2, pp. 686-695, 2007.

[15] P. Foomooljareon, W. A. C. Fernando, K. M. Ahmed, "PAPR reduction of OFDM systems using input sequence envelope scaling," IEEE Veh. Technol. Conference, Vol. 2, pp.1243, 2003.

[16] H. Ryu, J Lee, J Park, "Dummy Sequence Insertion (DSI) For PAPR Reduction In The OFDM Communication System", IEEE Transactions on Consumer Electronics, Vol.50, no.1, pp.89-94, 2004. 